\title{
Motion optimization and parameter identification for a human and lower-back exoskeleton model
}

\author{
Paul Manns $^{\dagger}$, Manish Sreenivasa ${ }^{\dagger}$, Matthew Millard ${ }^{\dagger}$ and Katja Mombaur ${ }^{\dagger}$
}

\begin{abstract}
Designing an exoskeleton to reduce the risk of lowback injury during lifting is challenging. Computational models of the human-robot system coupled with predictive movement simulations can help to simplify this design process. Here, we present a study that models the interaction between a human model actuated by muscles and a lower-back exoskeleton. We provide a computational framework for identifying the spring parameters of the exoskeleton using an optimal control approach and forward-dynamics simulations. This is applied to generate dynamically consistent bending and lifting movements in the sagittal plane. Our computations are able to predict motions and forces of the human and exoskeleton that are within the torque limits of a subject. The identified exoskeleton could also yield a considerable reduction of the peak lower-back torques as well as the cumulative lower-back load during the movements. This work is relevant to the research communities working on human-robot interaction, and can be used as a basis for a better human-centered design process.
\end{abstract}

Index Terms-Prosthetics and Exoskeletons, Optimization and Optimal Control, Physically Assistive Devices

\section{INTRODUCTION}

$\mathbf{L}$ OWER-back pain among workers in physically demanding jobs (e.g. nurses, airport baggage lifters, construction workers) is a frequent cause for absenteeism leading to production losses as well as burdens on the health infrastructure [1], [2]. In many of these work scenarios the load on the lower back, and the associated risk of injury, can be reduced by wearable robotic devices (exoskeletons) that support, augment or limit motion. Several challenges have to be solved before such devices become commonplace.

A majority of exoskeleton research and development focuses on the lower-limbs (e.g. [3]-[8]) or the shoulder-arm complex [9], [10]. Relatively few devices deal with movements of the back and torso. The movements and forces required to support the back require a specialized exoskeleton design. Some examples of exoskeletons developed for back support are Robomate [9], Laevo (Laevo B.V., Netherlands), PLAD [11] and WSAD [12]. The design of the exoskeleton may vary considerably, but in general, the idea is to support the extension moments at the back during bending thereby reducing the

Manuscript received: September, 10, 2016; Revised December, 21, 2016; Accepted February, 7, 2017.

This paper was recommended for publication by Ken Masamune upon evaluation of the Associate Editor and Reviewers' comments. Financial support by the European Commission within the H2020 project SPEXOR (GA 687662) is gratefully acknowledged.

${ }^{\dagger}$ The authors are with the Interdisciplinary of Scientific Computing, Heidelberg University, 69120 Heidelberg, Germany \{paul.manns, manish.sreenivasa, matthew.millard, kat ja.mombaur\}eiwr.uni-heidelberg. de

Digital Object Identifier (DOI): see top of this page.

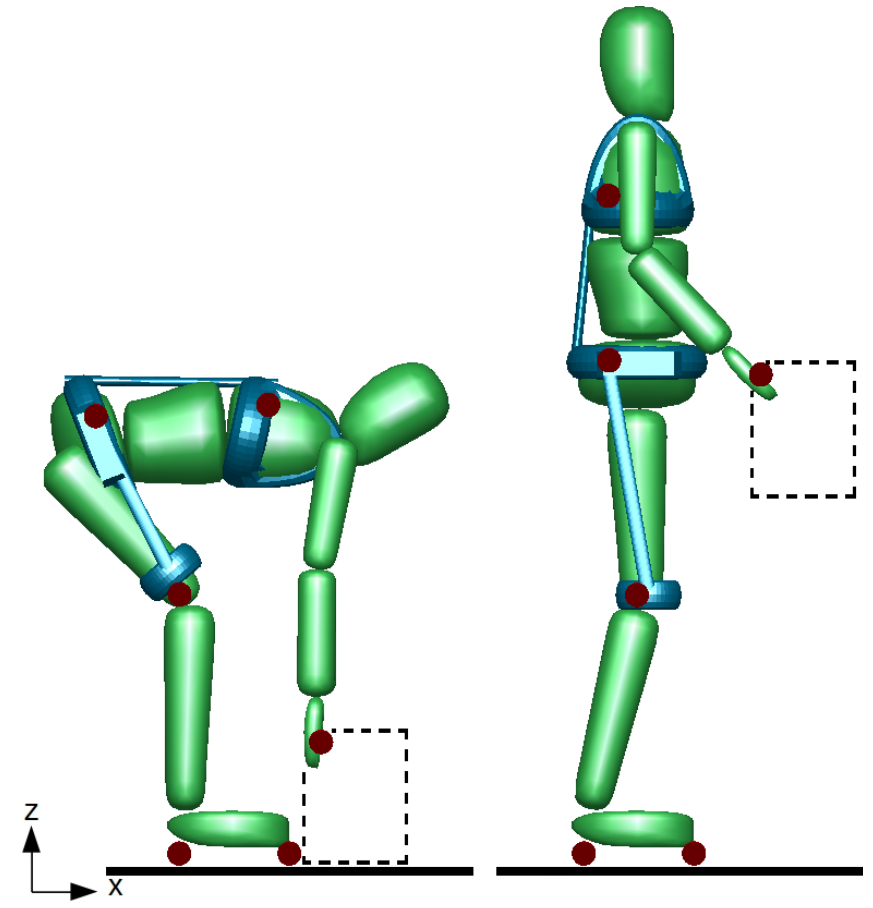

Fig. 1. Visualization of a human and exoskeleton model pose during a bending and lifting task. The attachment points of the exoskeleton on the thigh, pelvis and uppertrunk segments of the human body are marked with red circles. The red circles on the hands and feet indicate the contact points used for the lift, and the foot-ground contact points, respectively.

muscle forces as well as the corresponding vertebral forces. There is a gap in the experimental and simulation literature for studies that examine the motions and forces of supported human lifting. Additionally, there is limited knowledge on how we can predict lifting movements and the stresses this applies on the human body.

Here, we present a computational framework to help the design process and customization of lower-back exoskeletons to both the user and the type of movements they are intended to support. We chose a stoop motion (Fig. 1) for our case study because it is commonly used when picking something off the ground [13]. Back injury is correlated with the integral of net back moments over time [14], a quantity known as cumulative lower back load (CLBL), which we are able to compute with the presented framework. The study by Dieen et al [15] also found significant correlations between spinal compressive forces and the net moment about the L5/S1 vertebrae. Lowerback exoskeletons, such as the one modeled in our present study, aim to reduce the torque required during lifting motions, 
thereby reducing the risk of high compression forces and back injury.

Our work is in the context of a larger effort within the SPEXOR $^{1}$ project, aimed at developing a lower-back exoskeleton for vocational reintegration. The design of the exoskeleton in this project (as is likely the case for exoskeletons in general [10]) faces multiple challenges. The initial design parameters are only vaguely known and it can take many costly iterations before a design may be finalized. Furthermore, the vast range of human body shapes and potential movements makes it very labor-intensive to test all combinations experimentally.

Computational models of the human body and the exoskeleton can help with these issues and complement experimental testing. An important prerequisite is to predict the natural motion of the human body, while carrying our daily tasks such as lifting and walking. In this context our current study makes the following contributions:

- We develop a framework to model the effects of a passive lower-back exoskeleton worn by a human

- We identify the optimal exoskeleton parameters suited for a bending and lifting motion while carrying heavy loads (an object of $15 \mathrm{~kg}$ weight in our scenario)

For this initial work, we restrict ourselves to two-dimensional movements in the sagittal plane and a passive exoskeleton mechanism (i.e. only consisting of unactuated mechanical elements such as springs and dampers). It is important to note that our framework does not require any recorded motion data from the human subjects as input. Instead, the movement trajectories and the optimal exoskeleton parameters are solutions of an optimal control problem (OCP). OCPs, described further in Section III, allow to predict feasible motions for given dynamic models and suitable objective functions. They have been used successfully for robot and human motion generation in the past (e.g. [16]-[18]), and to a limited extent for the design of lower limb exoskeletons [19], [20].

\section{HumAN AND EXOSKELETON MODELING}

We model the human body in the sagittal plane as an articulated mechanism (Fig. 1). The dynamics governing this model may be described as:

$$
\begin{aligned}
M(\mathbf{q}) \ddot{\mathbf{q}}+c(\mathbf{q}, \dot{\mathbf{q}}) & =\tau+G(\mathbf{q})^{T} \lambda \\
g(\mathbf{q}) & =0,
\end{aligned}
$$

where $\mathbf{q}, \dot{\mathbf{q}}$ and $\ddot{\mathbf{q}}$ denote the generalized coordinates, their velocities and accelerations, $M$ the generalized inertia matrix, $c$ the Coriolis, gravitational and centrifugal forces and $\tau$ the impressed forces. $g$ denotes the scleronomic constraint function, $G$ its Jacobian and $\lambda$ the Lagrange multipliers. In our case, the generalized force vector consists of the summation of the agonist-antagonist forces exerted by muscle torque generators (MTGs), $\tau_{M}$, as well as the forces exerted by the exoskeleton $\tau_{E}$. The MTGs and the exoskeleton are described in the following sections. The forward dynamics is evaluated using Featherstone's articulated body algorithm (ABA) and spatial vector algebra as described in [21], [22]. We employ the

\footnotetext{
${ }^{1}$ www.spexor.eu
}

open source dynamics library Rigid Body Dynamics Library $(R B D L)$ [23], which implements ABA.

\section{A. Human body model}

Our human model is a kinematic tree consisting of 16 segments with a rotational joint between each segment. The pelvis is modeled with two additional translational degrees of freedom (DoFs) in the forward (x) and vertical (z) directions (Fig. 1). We use the anthropometric parameters of a 35 year old male subject weighing $77.5 \mathrm{~kg}$ with a height of $1.72 \mathrm{~m}$. The segment lengths, segment masses and inertia properties were computed based on the linear regression equations from De Leva [24].

Except for the DoFs at the pelvis, all the other 15 rotational joints of the model are actuated by pairs of agonist-antagonist MTGs, giving 30 actuators in total. The MTGs assume a rigid tendon and represent the overall torque being generated at a joint by the muscles in one rotational direction. In comparison to line-type muscles (see e.g. [25]), this simplification significantly reduces the computational time while preserving the experimentally measured torque-angle and torque-angularvelocity characteristics [26]-[28]. Our MTG model computes the joint torque as a function of joint angle, angular velocity, and muscle activation as

$$
\tau_{M}=\tau_{0}\left(a f^{A}(q) f^{V}(\dot{q})-\beta \frac{\omega}{\omega_{\max }}+f^{P}(q)\right)
$$

for either agonist or antagonist of a certain joint, where $\tau_{0}$ indicates the maximum isometric torque, $a$ denotes the normalized muscle activation ranging from 0 (no activation) to 1 (full activation), $f^{A}$ denotes the active torque-angle curve, $f^{V}$ denotes the active-torque-velocity curve and $f^{P}$ the passivetorque-angle curve. Damping is specified with the $\beta \omega / \omega_{\max }$ term. We have fitted the curves for $f^{A}, f^{V}, f^{P}$ as $5^{\text {th }}$ order Bezier splines. They are built on dynamometric data from the literature, primarily relying upon [26]-[28]. These curves are twice continuously differentiable which allows their use inside the OCP. The MTG implementation is available as open source software code ${ }^{2}$, with further details in [29].

\section{B. Exoskeleton model}

We model the exoskeleton as a torsion spring that applies a moment at the point $\mathrm{P}$ on the pelvis and attaches to the body and thigh at the points $\mathrm{T}$ and $\mathrm{U}$, respectively (Fig. 1, 2). We assume that the exo is securely fastened to the body and does not slip. The rigid bars that span the points P-T and P-U are fixed at point $\mathrm{P}$, but the ends are free to slide at the points $\mathrm{T}$ and $\mathrm{U}$. The torsion angle $\theta$ of the spring is given by

$$
\theta=(\pi-\alpha)-\theta_{0}
$$

where $\alpha$ is the angle extended between the points T, $\mathrm{P}$ and U. $\theta_{0}$ denotes the angle where the torsion spring is at rest. Considering an ideal spring, the resulting torque is

$$
\tau_{\text {spring }}=k \theta
$$

\footnotetext{
${ }^{2}$ https://bitbucket.org/rbdl/rbdl
} 


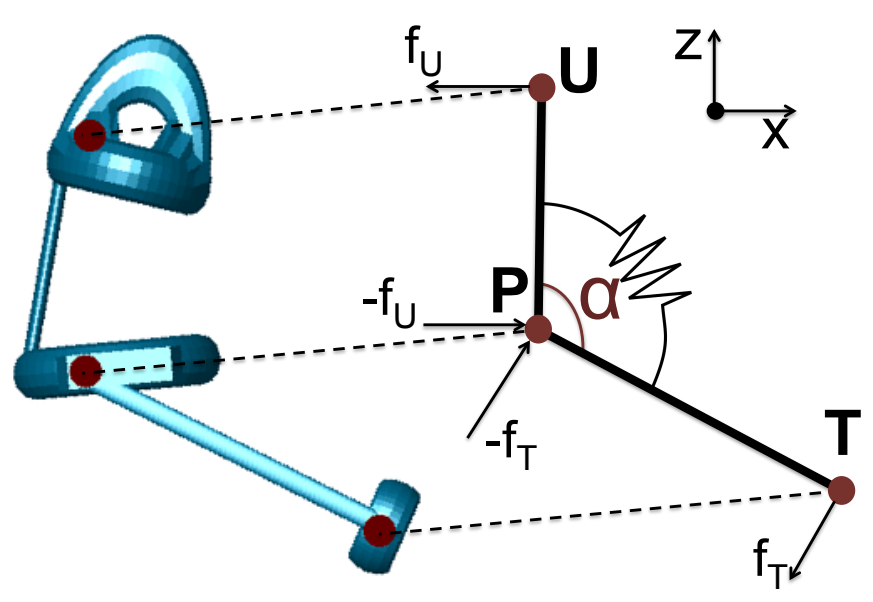

Fig. 2. Sketch of the exoskeleton mechanism in the sagittal plane. Forces and the directions they are acting in are marked with arrows. The attachment points are marked with filled red circles.

where $k$ denotes the stiffness of the spring. The resulting forces acting on the attachment points are given by $r \times f=\tau$. As the attachment points lie in the sagittal plane and the force acts perpendicular to the sliding bar on the attachment point, the forces acting on the attachment points can be computed as:

$$
\begin{aligned}
& f_{U}=\frac{\tau_{\text {spring }}}{\|\overrightarrow{P U}\|} \frac{\overrightarrow{P U} \times e_{y}}{\left\|\overrightarrow{P U} \times e_{y}\right\|} \\
& f_{T}=-\frac{\tau_{\text {spring }}}{\|\overrightarrow{P T}\|} \frac{\overrightarrow{P T} \times e_{y}}{\left\|\overrightarrow{P T} \times e_{y}\right\|}
\end{aligned}
$$

The Jacobian of the position of point A on the human model with respect to $\mathrm{q}$, the vector of generalized positions, is denoted $J(q, A)$. The generalized forces that arise from the exoskeleton mechanism are:

$$
\tau_{E}=J(\mathbf{q}, P)\left(-f_{T}-f_{U}\right)+J(\mathbf{q}, T) f_{T}+J(\mathbf{q}, U) f_{U}
$$

The computation for the spring mechanism on the other side of the body is identical. The resulting generalized torque $\tau_{E}$ is added to the torque vector arising from the MTGs before the sum is applied to the rigid body model.

The angle $\theta_{0}$ and the spring stiffness $k$ make up the two design parameters that are to be identified in the OCP. We set angular limits such that physiologically unrealistic extension of the body is not possible. This is the same as assuming that the exoskeleton is physically limited by hardstops in extension. This is necessary because making $\theta_{0}$ subject to the optimization can yield a preloaded spring generating an extension moment when standing straight.

We assume an exoskeleton mass of $6.6 \mathrm{~kg}$ similar to existing passive exoskeletons [30]. We distribute this mass to the pelvis, left thigh, right thigh, middletrunk and uppertrunk segments with the ratios $1 / 2,1 / 8,1 / 8,1 / 8$ and $1 / 8$ respectively. Note that in this first approach we add the exoskeleton mass to the body segments, and ignore some of the mechanical effects and restrictions the exoskeleton imposes on the motion.

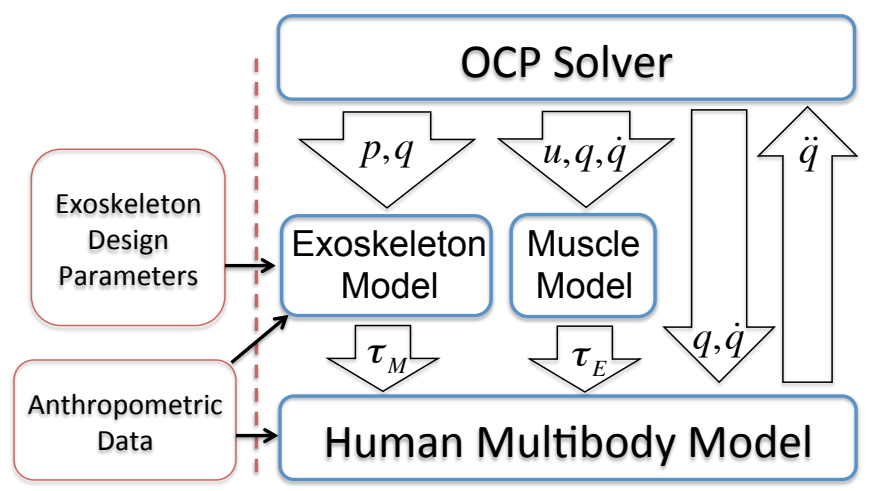

Fig. 3. Overview of the OCP setup: The differential state vector of the OCP consists of the generalized coordinates $\mathbf{q}$ and the corresponding velocities $\dot{\mathbf{q}}$. The controls are denoted $\mathbf{u}$ and the global parameters are denoted p. $\tau_{M}$ are the generalized forces from the muscle torque generators and $\tau_{E}$ the generalized forces arising from the exoskeleton model.

\section{MOTION AND PARAMETER OPTIMIZATION}

We formulate our OCP (overview in Fig. 3) as a $N$ phase problem of the form:

$$
\min _{\mathbf{x}, \mathbf{u}, \mathbf{p}, \mathbf{T}} \sum_{i=0}^{N-1} \int_{T_{i}}^{T_{i+1}} \phi_{i}(\mathbf{x}(t), \mathbf{u}(t), \mathbf{p}) \mathrm{d} t
$$

s.t.

$$
\begin{aligned}
\dot{\mathbf{x}}= & f_{i}(\mathbf{x}(t), \mathbf{u}(t), \mathbf{p}), t \in\left[T_{i}, T_{i+1}\right] \\
0 \leq & g_{i}(\mathbf{x}(t), \mathbf{u}(t), \mathbf{p}), t \in\left[T_{i}, T_{i+1}\right] \\
0= & h_{i}(\mathbf{x}(t), \mathbf{u}(t), \mathbf{p}), t \in\left[T_{i}, T_{i+1}\right] \\
0 \leq & r_{i e q}\left(\mathbf{x}\left(T_{0}\right), \ldots, \mathbf{x}\left(T_{N-1}\right), \mathbf{p}\right) \\
0= & r_{e q}\left(\mathbf{x}\left(T_{0}\right), \ldots, \mathbf{x}\left(T_{N-1}\right), \mathbf{p}\right) \\
& 0=T_{0}<\ldots<T_{N-1}=T_{f}
\end{aligned}
$$

The differential states of the system are denoted $\mathbf{x}$, the controls are denoted $\mathbf{u}$, and the global parameters are denoted $\mathbf{p}$. The global parameters $\mathbf{p}$ allow us to incorporate the exoskeleton design parameters $\theta_{0}$ and $k$. The variables $T_{i}, i \in\{0, \ldots, N-1\}$ are the start and end times of the different phases and also subject to the optimization. Equation (9) is the minimization objective $\phi_{i}$. The function $f_{i}$ in Eq. (10) describes the system dynamics. Our human model has a floating base and consequently, we impose equality constraints $h_{i}$ modeling the heel and toe positions which are kept in contact with the floor throughout the motion. In addition, we impose inequality constraints $g_{i}$ to maintain positive contact forces at these points. Constraints at the start, lift and end points such as the hand positions in task space enter the boundary constraints $r_{i e q}$ and $r_{e q}$. Note that our system is scleronomous and hence, $f_{i}$, $g_{i}$ and $h_{i}$ do not depend on $t$ explicitly.

The problem described above contains significant nonlinearities and is hard to solve. The nonlinearities stem from the computation of the generalized inertia matrix, the MTG computations and the constraints taking care of avoiding interpenetrations. In particular, the set of feasible solutions is non-convex in general and we can only expect to obtain a local solution when computing the solution numerically. We use a direct solution approach that discretizes the controls in 
the time domain as piecewise linear functions. Direct multiple shooting [31] is a popular approach for solving such problems. It is based on a subdivision of the phases into shooting intervals on which initial value problems of the ordinary differential equations are solved depending on the current state, control and parameter iterate. We employ the SQP-based direct multiple shooting implementation MUSCOD-II [32] to solve the OCPs described here.

\section{A. Objective function}

For simulating the motions in this work we use an objective function that minimizes the squared muscle activations over time. This formulation is commonly used in literature on optimal control for human motion generation and is associated with the minimization of muscle effort [33]. In our case the muscle activations a are the controls $\mathbf{u}$ of the OCP, hence we may formulate our objective function as:

$$
\min \sum_{i=0}^{N-1} \int_{T_{i}}^{T_{i+1}} \mathbf{u}(t) \cdot \mathbf{u}(t) \mathrm{d} t
$$

\section{B. Movement scenarios}

The movement we consider for the trajectory optimization consists of $N=2$ phases, namely bending and lifting. The human model starts from an upright pose while standing still (all velocities to zero). At the start of the second phase the model's hands are constrained to be at a lifting point directly in front and close to the ground. At the end of the second phase the model is required to be in an upright pose while standing still. We introduce a slight left-right asymmetry in the initial pose and the lift point as in real human movements perfect symmetry is unlikely. This allows the optimization routine to find non-symmetric solutions which would be not possible otherwise. We compute four movements: $0 \mathrm{~kg}$ and $15 \mathrm{~kg}$ lift both with and without exoskeleton. The $0 \mathrm{~kg}$ lift was chosen because many agricultural workers pick fresh crops and suffer from back pain [34]. The $15 \mathrm{~kg}$ lift was chosen because it is in the middle of the heavy category for frequent lifting [35]. Importantly, this category of workers experience significantly more back pain than workers in the medium category.

\section{Multiple Shooting Setup}

We discretize both phases with 25 shooting nodes and 25 control intervals. Both phases are initialized with a duration of $1 \mathrm{~s}$. The start and end configuration of the model for both phases are initialized with predefined feasible poses in upright and bent position. The intermediate trajectories are then initialized as a linear interpolation between the given poses. All joint velocities are initialized as 0 . For all control trajectories, we have chosen piecewise linear, globally continuous splines. They are initialized with a muscle activation of 0.25 for the whole time horizon.

\section{RESUlTS}

Each of the four OCPs took between 4 to 8 hours to solve on a standard desktop computer (Intel(R) Core(TM) i7-4790K CPU with $4.00 \mathrm{GHz}$ running with 4 compute threads).
TABLE I

SWITCHING AND FINAL TIME OF THE OPTIMIZED MOTION FOR 0 KG AND 15 KG LIFTS WITH AND WITHOUT EXOSKELETON ASSIST

\begin{tabular}{|r||c|c|c|c|}
\hline & 0 kg no exo & 0 kg exo & $15 \mathrm{~kg}$ no exo & $15 \mathrm{~kg}$ exo \\
\hline \hline$T_{\text {switch }}$ & 0.80 & 0.98 & 0.99 & 0.80 \\
$T_{\mathrm{f}}$ & 2.00 & 2.18 & 2.15 & 1.79 \\
\hline
\end{tabular}

\section{A. Movement Profiles}

For all cases the predicted movements showed smooth profiles and stayed within the limits defined for joint angles, joint angular velocities as well as the generated muscle torques. We observed that the lumbar angle $\beta$, the angle between the pelvis and the upper trunk, showed greater variation with the exoskeleton for the $15 \mathrm{~kg}$ lift, than while lifting no weight (Fig. 4). The maximum angular difference between the lumbar angles was $11.2^{\circ}$, the one at the switching point was $0.81^{\circ}$, the difference between peak lumbar angles was $1.25^{\circ}$. The durations of the movements differed depending on the lifting weight as well as whether the exoskeleton assist was available or not (see Table I). The largest time difference was found for the $15 \mathrm{~kg}$ lift, which lasted $2.15 \mathrm{~s}$ without the exoskeleton and $1.79 \mathrm{~s}$ with the exoskeleton.

\section{B. Lumbar extension torque and cumulative load}

The exoskeleton assist considerably reduces the lower-back extension torques exerted by the model (Fig. 5). The peak torque was lowered from $271.6 \mathrm{Nm}$ to $29.5 \mathrm{Nm}$ in the $0 \mathrm{~kg}$ case, and from $358.3 \mathrm{Nm}$ to $112.3 \mathrm{Nm}$ in the $15 \mathrm{~kg}$ case. The associated muscle effort (as measured by the objective function value) required for the lift also reduced significantly when the model was wearing an exoskeleton; by $76.4 \%$ in the $0 \mathrm{~kg}$ case and by $51.9 \%$ in the $15 \mathrm{~kg}$ case. CLBL reduced by $93.8 \%$ from $117.54 \mathrm{Nms}$ to $7.29 \mathrm{Nms}$ for the $0 \mathrm{~kg}$, and by $79.28 \%$ from $222.14 \mathrm{Nm} \mathrm{s}$ to $46.02 \mathrm{Nm} \mathrm{s}$, for the $15 \mathrm{~kg}$ case.

\section{Optimal exoskeleton parameters}

The optimal spring offset angle, $\theta_{0}$, were found to be $-8.88^{\circ}$ and $-9.28^{\circ}$ for the $0 \mathrm{~kg}$ and $15 \mathrm{~kg}$ lift, respectively. Note that for our setup, $\theta_{0}=5.9^{\circ}$ would yield a completely unloaded spring when standing upright. The OCP results therefore favor preloading the spring to generate small extension moments when the human is standing upright. The optimized spring stiffness for the $0 \mathrm{~kg}$ lift was found to be $72.1 \mathrm{Nm} / \mathrm{rad}$ and that for the $15 \mathrm{~kg}$ lift was $31 \%$ lower at $50.0 \mathrm{Nm} / \mathrm{rad}$.

\section{DISCUSSION}

In this study we have presented a framework to model the interaction between a human and a lower-back exoskeleton. Additionally, we predict the movements during a bending and lifting task using an optimal control approach and identify the exoskeleton spring parameters for these movements. Our results provide the first steps towards applying model-based predictive simulations of human-robot interaction to design wearable robotic devices. To the best of our knowledge such approaches are not yet widely used with few comparable 

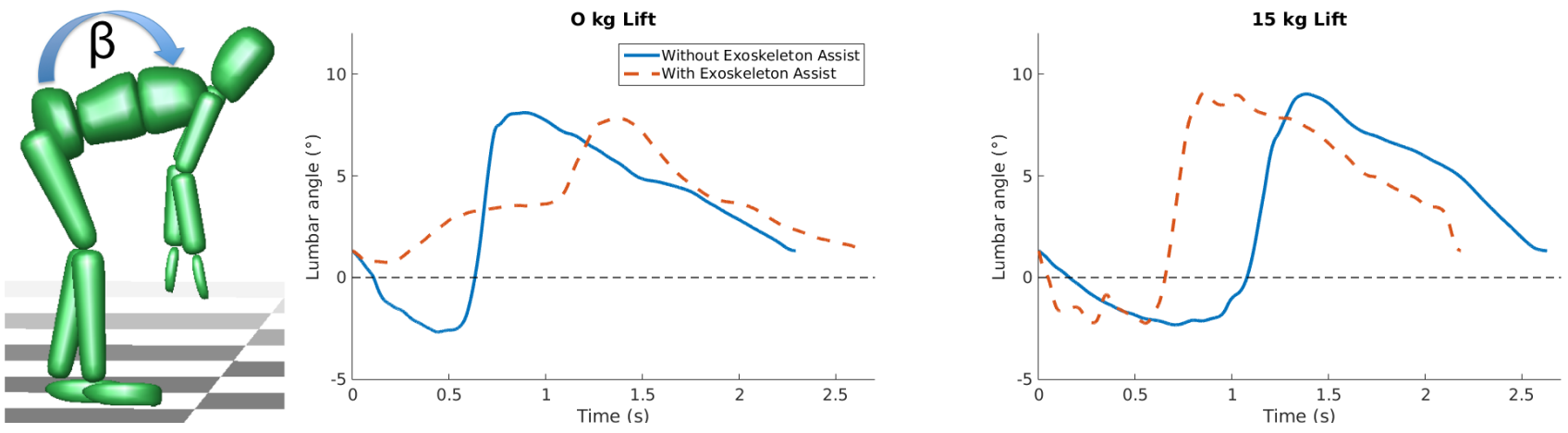

Fig. 4. Progression of lumbar angle, $\beta$, during the movements with and without exoskeleton assist for $0 \mathrm{~kg}$, and $15 \mathrm{~kg}$ lifts.
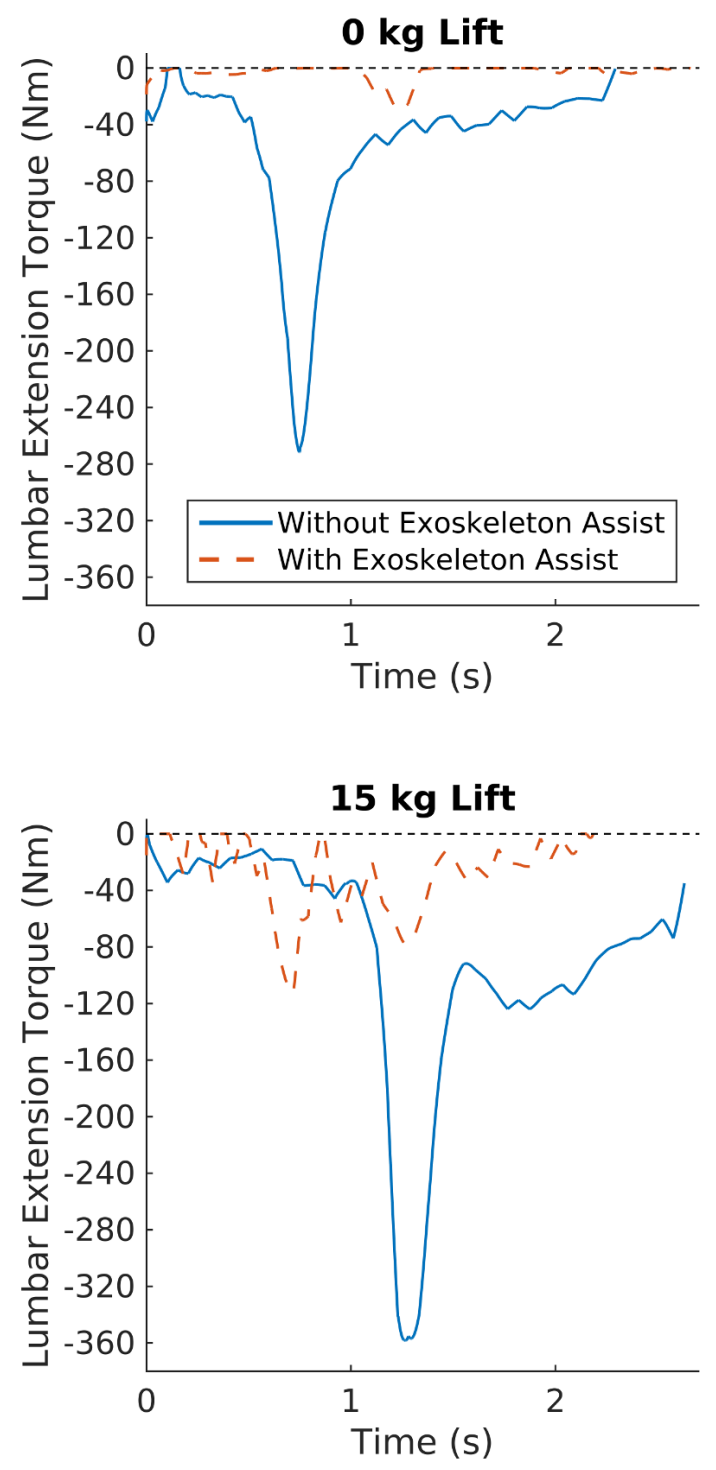

Fig. 5. Profile of the muscle extension torques at the lower back with and without exoskeleton assist for (a) $0 \mathrm{~kg}$ and (b) $15 \mathrm{~kg}$ lifts. studies (see e.g. [19], [20], [36], [37]). We note the following important points about our present work:

- The motion computation requires minimal input about the human operator (height and weight), pre-defined motions (kinematic pose at start, lift and end), as well as the exoskeleton design. This aspect can help to reduce the time and effort required in the initial design phases.

- The OCP framework produces (predicts) dynamically consistent motions for the whole simulation. With the use of MTGs for the human, and feasible mechanical models of the exoskeleton, the results are also physiologically meaningful.

To transfer our approach to the exoskeleton design process; we may imagine that a large number of such simulations would be carried out on a variety of tasks, lifting weights, human body proportions as well as exoskeleton designs. The result of this process may then be one or several exoskeleton designs that are best able to satisfy the design requirements (e.g. reduce lower-back torques by $40 \%$ ).

Our results show that the OCP is able to identify exoskeleton parameters that reduced both the peak torques at the lowerback and CLBL. This reduction is desirable as high peak lower-back torques and CLBL is associated with lower-back pain and injury [14], [15]. Note that both of these quantities were not tied to the chosen objective function. Comparing to literature, our simulations yielded peak back extension torques that were higher than those reported in [38] $(221 \pm 24 \mathrm{Nm}$ for a $10 \mathrm{~kg}$ lift). As well, the flexion angle reported in [38] $\left(39 \pm 15^{\circ}\right)$ is higher than the ones in our model $\left(8-9^{\circ}\right)$. We expect that appropriate changes to the cost function could align our simulation results more closely with those from [38]. The reason for these differences is likely because we treated muscle activation as being equally expensive across all muscles. If the activation of the back muscles were to be weighted as more expensive, relative to other muscles, the lumbar spine would go into deeper flexion. As the variation in lumbar flexion angle can be quite variable $\left(\sigma=15^{\circ}[38]\right)$ the weighting pattern likely varies from one subject to the next. The peak lumbar torque can be reduced by including the total torque the muscle generates in the cost function. Under an activation squared cost function the passive forces of the hip extensors (which the solution exploits) are without cost. Inverse optimal control can provide means to identify the cost function and weighting 
terms from experimental data [20], and is one of avenues we are currently exploring.

The optimal parameters of the exoskeleton varied with the weight being lifted as did the value of the cost function. As expected the exoskeleton assisted motion resulted in a large reduction in the cost function value. Interestingly, the heavier weight yielded a less stiff spring while the rest lengths were similar for both $0 \mathrm{~kg}$ and $15 \mathrm{~kg}$ lifts. The $15 \mathrm{~kg}$ lift requires a lighter spring because the passive component of the hip extensors are being exploited to carry more of the load. This observation has important ramifications: the ideal spring coefficent for a subject is likely influenced by the flexbility of their hips. Since flexbility varies greatly from one person to the next, it is important that the stiffness characteristics of the exoskeleton be easily adjusted. Interestingly, the time of the motion with and without exoskeleton showed different trends for $0 \mathrm{~kg}$ and $15 \mathrm{~kg}$ weight. The reason for these differences are presently unclear and may be related to the cost function formulation as well as OCP solver exploiting the cost-free passive muscle forces. These issues are the subject of further investigation, as well as comparisons to experimental lifting data.

\section{Limitations and Perspectives}

This work is an initial approach towards model-based design of spinal exoskeletons. To focus on the development of the framework we have introduced several model simplifications that must be taken into consideration. Here we list some of these limitations, their possible influence on our results, and perspectives towards improving our current framework in this context.

The objective function used in this study is typically used for the generation of gait [26]. The extent to which this is applicable to lifting motions is presently unclear. Additionally, neither our current cost function, nor the constraints, account for ergonomic issues such as comfort and pain (e.g. from pressure at contact points). Incorporating an ergonomically oriented component in the OCP is an important next step towards designing exoskeletons that will be acceptable by the end-users.

Personalization of the human and exoskeleton model to the user is another vital requirement. Here we developed a human model (and the corresponding exoskeleton) scaled to the proportions of our single test subject. In current work we are developing methods to identify the body parameters from experimental data, in order to better match the body proportions and physical strengths of a range of subjects.

The movements limited to the sagittal plane and our simplified exoskeleton model provide only a gross approximation of the real-world lifting scenarios. While our current work illustrates the potential of optimal control methods for exoskeleton design, further developments are necessary to make them usuable in a general sense. For example, extending the human and exoskeleton models to movements in the transverse and coronal planes, and, modeling the exoskeleton as an external kinematic chain coupled to the body at the contact points. Finally, these perspectives should be complemented with validation of the methodology including experimental trials with a cohort of healthy subjects lifting with and without an exoskeleton.

\section{ACKNOWLEDGMENT}

The authors would like to thank the Simulation and Optimization research group of H.G. Bock at Heidelberg University, Germany for allowing us to use the software MUSCODII.

\section{REFERENCES}

[1] K. B. Hagen and O. Thune, "Work incapacity from low back pain in the general population," Spine, vol. 23, no. 19, pp. 2091-2095, 1998.

[2] N. Maniadakis and A. Gray, "The economic burden of back pain in the UK," Pain, vol. 84, no. 1, pp. 95-103, 2000.

[3] A. B. Zoss, H. Kazerooni, and A. Chu, "Biomechanical design of the Berkeley lower extremity exoskeleton (BLEEX)," IEEE/ASME Trans. On Mechatronics, vol. 11, no. 2, pp. 128-138, 2006.

[4] M. Talaty, A. Esquenazi, and J. E. Briceño, "Differentiating ability in users of the ReWalk(TM) powered exoskeleton: An analysis of walking kinematics," in Rehabilitation Robotics (ICORR), 2013 IEEE Int. Conf. on. IEEE, 2013, pp. 1-5.

[5] S. A. Kolakowsky-Hayner, J. Crew, S. Moran, and A. Shah, "Safety and feasibility of using the Ekso(TM) bionic exoskeleton to aid ambulation after spinal cord injury," Journal of Spine, vol. 2013, 2013.

[6] J. Moreno, G. Asin, J. Pons, H. Cuypers, B. Vanderborght, D. Lefeber, E. Ceseracciu, M. Reggiani, F. Thorsteinsson, A. del Ama, et al., "Symbiotic wearable robotic exoskeletons: the concept of the Biomot project," in Int. Workshop on Symbiotic Interaction. Springer, 2014, pp. 72-83.

[7] J. Beil, G. Perner, and T. Asfour, "Design and control of the lower limb exoskeleton KIT-EXO-1," in 2015 IEEE Int. Conf. on Rehabilitation Robotics (ICORR). IEEE, 2015, pp. 119-124.

[8] A. Wall, J. Borg, and S. Palmcrantz, "Clinical application of the hybrid assistive limb (HAL) for gait training - a systematic review," Frontiers in Systems Neuroscience, vol. 9, 2015.

[9] K. Stadler, W. Elspass, and H. van de Venn, "Robo-mate: Exoskeleton to enhance industrial production," Mobile Service Robotics: CLAWAR 2014, vol. 12, p. 53, 2014.

[10] E. Rocon and J. L. Pons, Exoskeletons in rehabilitation robotics - Tremor Suppression. Springer Tracts in Advanced Robotics, 2011.

[11] M. Abdoli-E, M. J. Agnew, and J. M. Stevenson, "An on-body personal lift augmentation device (PLAD) reduces EMG amplitude of erector spinae during lifting tasks," Clinical Biomechanics, vol. 21, no. 5, pp. 456-465, 2006

[12] Z. Luo and Y. Yu, "Wearable stooping-assist device in reducing risk of low back disorders during stooped work," in 2013 IEEE Int. Conf. on Mechatronics and Automation. IEEE, 2013, pp. 230-236.

[13] J. H. van Dieën, M. J. Hoozemans, and H. M. Toussaint, "Stoop or squat: a review of biomechanical studies on lifting technique," Clinical Biomechanics, vol. 14, no. 10, pp. 685-696, 1999.

[14] P. Coenen, I. Kingma, C. R. Boot, J. W. Twisk, P. M. Bongers, and J. H. van Dieën, "Cumulative low back load at work as a risk factor of low back pain: a prospective cohort study," Journal of occupational rehabilitation, vol. 23, no. 1, pp. 11-18, 2013.

[15] J. Van Dieën and I. Kingma, "Effects of antagonistic co-contraction on differences between electromyography based and optimization based estimates of spinal forces," Ergonomics, vol. 48, no. 4, pp. 411-426, 2005.

[16] J. E. Bobrow, S. Dubowsky, and J. Gibson, "Time-optimal control of robotic manipulators along specified paths," The Int. Journal of Robotics Research, vol. 4, no. 3, pp. 3-17, 1985.

[17] O. von Stryk and M. Schlemmer, "Optimal control of the industrial robot Manutec r3," in Computational Optimal Control. Springer, 1994, pp. $367-382$.

[18] K. Mombaur, A. Truong, and J.-P. Laumond, "From human to humanoid locomotion - an inverse optimal control approach," Autonomous robots, vol. 28 , no. 3, pp. 369-383, 2010.

[19] H. Koch and K. Mombaur, "ExoOpt - a framework for patient centered design optimization of lower limb exoskeletons," in 2015 IEEE Int. Conf. on Rehabilitation Robotics (ICORR). IEEE, 2015, pp. 113-118. 
[20] K. Mombaur, "Optimal control for applications in medical and rehabilitation technology: Challenges and solutions," in Advances in Mathematical Modeling, Optimization and Optimal Control. Springer, 2016, pp. 103-145.

[21] R. Featherstone, "The calculation of robot dynamics using articulatedbody inertias," The Int. Journal of Robotics Research, vol. 2, no. 1, pp. 13-30, 1983.

[22] — Rigid body dynamics algorithms. Springer, 2014.

[23] M. L. Felis, "RBDL: An efficient rigid-body dynamics library using recursive algorithms," Autonomous Robots, pp. 1-17, 2016.

[24] P. De Leva, "Adjustments to Zatsiorsky-Seluyanov's segment inertia parameters," Journal of Biomechanics, vol. 29, no. 9, pp. 1223-1230, 1996.

[25] S. L. Delp, F. Anderson, A. Arnold, P. Loan, A. Habib, C. John, E. Guendelman, and D. G. Thelen, "Opensim: Open-source software to create and analyze dynamic simulations of movement," IEEE Trans. in Biomedical Engineering, vol. 54, pp. 1940-1950, 2007.

[26] D. E. Anderson, M. L. Madigan, and M. A. Nussbaum, "Maximum voluntary joint torque as a function of joint angle and angular velocity: model development and application to the lower limb," Journal of Biomechanics, vol. 40, no. 14, pp. 3105-3113, 2007.

[27] M. I. Jackson, "The mechanics of the table contact phase of gymnastics vaulting," Ph.D. dissertation, Loughborough University, 2010.

[28] B. B. Kentel, M. A. King, and S. R. Mitchell, "Evaluation of a subjectspecific, torque-driven computer simulation model of one-handed tennis backhand ground strokes," Journal of Applied Biomechanics, vol. 27, no. $4,2011$.

[29] M. Millard, M. Sreenivasa, P. Manns, and K. Mombaur, "Predicting human-machine interaction: How much human-model detail is necessary?" in IEEE Int. Conf. on Robotics and Automation (ICRA), submitted.

[30] Y. Ikeuchi, J. Ashihara, Y. Hiki, H. Kudoh, and T. Noda, "Walking assist device with bodyweight support system," in 2009 IEEE/RSJ Int. Conf. on Intelligent Robots and Systems. IEEE, 2009, pp. 4073-4079.

[31] H. G. Bock and K.-J. Plitt, "A multiple shooting algorithm for direct solution of optimal control problems." Proceedings Of The IFAC World Congress, 1984.

[32] D. B. Leineweber, I. Bauer, H. G. Bock, and J. P. Schlöder, "An efficient multiple shooting based reduced SQP strategy for large-scale dynamic process optimization. Part 1: Theoretical aspects," Computers \& Chemical Engineering, vol. 27, no. 2, pp. 157-166, 2003.

[33] M. Ackermann and A. J. van den Bogert, "Optimality principles for model-based prediction of human gait," Journal of Biomechanics, vol. 43, no. 6, pp. 1055-1060, 2010.

[34] F. A. Fathallah, "Musculoskeletal disorders in labor-intensive agriculture," Applied ergonomics, vol. 41, no. 6, pp. 738-743, 2010

[35] V. Mooney, K. Kenney, S. Leggett, and B. Holmes, "Relationship of lumbar strength in shipyard workers to workplace injury claims," Spine, vol. 21, no. 17, pp. 2001-2005, 1996.

[36] F. Mouzo, U. Lugris, J. Cuadrado, J. M. Font-Llagunes, and F. J. Alonso, "Evaluation of motion/force transmission between passive/active orthosis and subject through forward dynamic analysis," in Int. Conf. on Neuro-Rehabilitation (ICNR), 2016.

[37] S. Wang, W. van Dijk, and H. van der Kooij, "Spring uses in exoskeleton actuation design," in Int. Conf. on Rehabilitation Robotics (ICORR), June 2011, pp. 1-6.

[38] I. Kingma, T. Bosch, L. Bruins, and J. H. van Dieën, "Foot positioning instruction, initial vertical load position and lifting technique: effects on low back loading," Ergonomics, vol. 47, no. 13, pp. 1365-1385, 2004. 